\title{
ENABLING NETWORK CONVERGENCE THROUGH CONTEXTUAL SESSION MOBILITY WITH IMS
}

\author{
A. Dragoi ${ }^{1}$, N. Kara ${ }^{2}$ and S. Aissa ${ }^{3}$ \\ ${ }^{1}$ Department of Computer Science, University of Waterloo, Waterloo, CA \\ oadragoiealumni.uwaterloo.ca \\ ${ }^{2}$ Department of Software and IT Engineering, ETS University of Quebec, Montreal, CA \\ Nadjia.Kara@etsmtl.ca \\ ${ }^{3}$ Department of Telecommunication, INRS-EMT University of Quebec, Montreal, CA \\ aissa@emt.inrs.ca
}

\begin{abstract}
To fully benefit from the all IP convergence, it should be possible for a user not only to access services ubiquitously and at any time, but also to automatically and seamlessly transfer ongoing communication between different devices. This article investigates the problem space of supporting Session Mobility (SM) with IMS and the associated issues such as when and how to support dynamic changes in the set of devices through which the user accesses a session. It also investigates how the SM arrival calls should be handled at the network level where they are not considered as ongoing sessions. Hence, we discuss the design options and identify functional blocks needed to support SM. We propose applying priority-based resource allocation approach to guarantee lower blocking probability for ongoing SM arrival calls compared to new arrival calls. Numerical results show that this approach allows achieving such performance for different call arrival rates.
\end{abstract}

KEYWORDS

Session mobility; IMS; context aware; mobile networks; service performance.

\section{INTRODUCTION}

Mobile services access is growing at the expense of fixed ones. Mobile terminals and tariffs are becoming more affordable and now the users are calling a person and not a location. Consequently, the migration to fixed mobile converged network infrastructure is gaining more attention by the network manufacturers and operators. The fixed mobile convergence concept aims to develop an infrastructure that allows users to access to telecommunication services irrespective of the access technology used. Users could access a set of multimedia services through a variety of access technologies such as $3 \mathrm{G}$, WiFi, Twisted pair and coax. IP Multimedia Subsystem (IMS) architecture has been proposed by the 3GPP standard to provide convergence of mobile and fixed networks through an all IP infrastructure [1]. The convergence can be achieved by coordinating the concepts, the functions and the capabilities supported by the fixed and the mobile networks.

Mobility, in all its facets (terminal, domain, service, personal and session mobility) has been widely investigated, and mobility schemes have been proposed at the network, transport, and application layers. IETF RFCs discuss SIP (Session Initiation Protocol) mechanisms to implement the transfer of the end point of a multimedia communication from one device to another [2]. Academic research further investigates session mobility (SM), but it overlooks some IMS integration issues, such as how and why a session end-points are transferred, and how to discover, identify and authorize devices [3][4][5][6]. Implementation attempts are limited to trials and evaluation of the delays. Ubiquitous and pervasive computing research

DOI : 10.5121/ijma.2011.3202 
The International Journal of Multimedia \& Its Applications (IJMA) Vol.3, No.2, May 2011

community did study such integration aspects, but not within an IMS framework. Instead, proprietary distributed environments are developed spanning devices and so-called smart rooms and spaces.

Mobility management is at the heart of the convergence. It could be supported at the application and at the network level. The challenge in supporting the SM is to improve user's quality of experience (QoE). Many wired and wireless devices are increasingly used concurrently and interdependently. The QoE can be improved through support for dynamic changes in the set of devices through which users access a session, with seamless transfer of on-going communication between select devices, as the context in which users evolve changes. It can also be improved by guaranteeing network resource availability while moving or splitting the session media flows across different user equipments (UEs). The network resources allocation issue has been widely investigated to support the UEs move through mobile networks but has not been considered so far for SM. For the UEs mobility, Mobile IP (MIP) and several channel assignment approaches have been proposed respectively in the wired network and the radio access parts to guarantee an efficient delivery of multimedia flows. For the SM, Mobile SIP strategies have been added to ensure session continuity at the application level. However, the $\mathrm{SM}$ in the radio access part is seen as a new call arrival which will have less priority than a handoff call arrival. The target UE requesting a radio channel to carry the transferred session should have greater priority than that carrying a new call because the transferred session is an on going communication. It could have the same priority as a handoff or less depending on the probability needed for the handoff and the SM call arrivals failures. Hence, new call arrivals and call arrivals carrying transferred sessions should be handled differently at the network level. This article investigates what it takes to implement generic mechanisms for contextual SM with IMS, to help developers implement innovative session-related services. It also proposes and evaluates the usage of a priority-based radio resource allocation approach to support SM at the network level.

The remainder of this paper is organized as follows. Section 2 details the terminology and the notions used in this article. Section 3 reviews the current state of the art. Section 4 explores the design options in supporting contextual session-transfer with IMS and identifies functional blocks needed to implement such mechanisms at the level of a Session Controller Application Server. Section 5 describes a three dimensional priority-based radio resource allocation approach to manage the new, the handoff and the SM call arrivals in mobile converged networks. An analytic model is described in section 6 that allows computation of the blocking probability of the different calls. Section 7 discusses the numerical results. Conclusion is given in section 8 .

\section{SESSION MOBILITY}

Value-added custom session-services that capitalize on SM can be offered by an IMS operator in conjunction with a specific session-account, with the possibility to charge for such services. Such services would act as listener of the signalling traffic, but will intervene in the traffic to trigger and implement reconfigurations of an on-going user session to improve the QoE. In that, they differ from typical telecommunication services which would normally intervene only during the set-up or the tear-down of a call leg. Several human users will be associated with a session-account, just like several users are associated with a family plan in the commercial offers currently available on the market from cellular operators. The notion of account, as defined here, roughly corresponds to the notion of subscription, which is defined by 3GPP in [7] as the "description of the commercial relationship between the subscriber and the service providers". 
For each such an account one can formalize the notion of session as the collection of in-use endpoints, their respective states, as they are perceived at the network/operator level, and all the communication to/from the in-use end-points to corresponding end-points(figure 1).

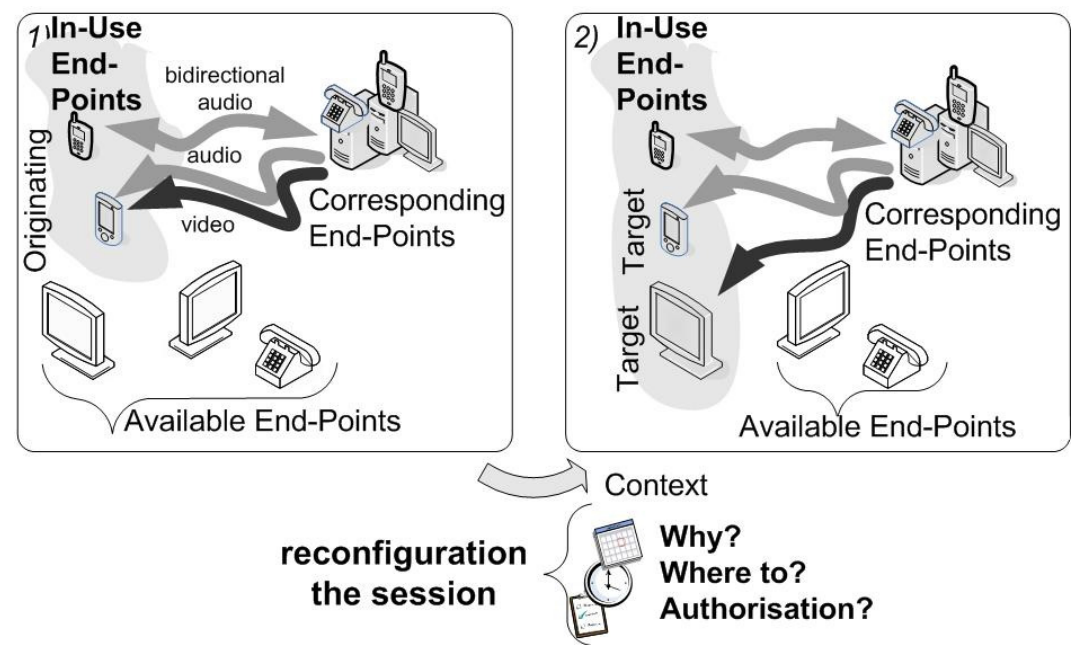

Figure 1. Seamless session-mobility

This notion of session is somewhat related to both the notion of group call, defined by 3GPP in [7] as "the relationship that exists between the members of a group for the purpose of transferring data" and to the generic notion of IMS session, defined in [1] as "a set of multimedia senders and receivers and the data streams flowing from senders to receivers". However, as defined in this article, the notion of session refers to a collection of end-points belonging to a user and all associated with an account. These end-points communicate with a set of corresponding end-points, not associated with that account.

An end-point is either a physical device, such as a cellular phone with a built-in IMS private identity and a public identity, or any piece of software registering as a client with IMS, such as a program on a PDA, a laptop or a TV set-top box. An in-use end-point is any end-point belonging to a human user associated with the account and that is currently involved in communication. A corresponding end-point is any other end-point to which one of the in-use end-points communicates, such as other devices of other users, content servers, and application servers. In a simple definition, a communication is any media stream set up over SIP. In a more generic definition however, a communication can be any other interaction of which the operator can be aware and that can be uniquely identified (e.g., media streams, sequences of HTTP requests/replies), regardless of how it was initiated (e.g., SIP, HTTP, RTSP). Considering only communication of which the operator/network can be aware makes it possible to implement the custom session-related functionality inside the network.

The notion of mobility of such a session refers to the operation of replacing one or more of the in-use end-points (the originating end-points) with one or more end-points (the target endpoints) from a set of available end-points, while maintaining unchanged the corresponding endpoints and the on-going communication, with adaptation to the different network and terminal characteristics, if needed. This should happen without the need for users to manually reconfigure each communication stream. Figure 1 gives an example. Initially, a cell phone and a PDA are in-use. The originating end-point is the PDA. The cell phone and the PDA remain inuse. The uni-directional video stream originally directed to the PDA is redirected towards the nearby display, to capitalize on its greater screen size, but the associated audio track remains 
The International Journal of Multimedia \& Its Applications (IJMA) Vol.3, No.2, May 2011

directed to the PDA. The conversational bidirectional voice communication remains unchanged, and so do the corresponding nodes: the video server streaming video and audio and the peer in the voice conversation.

The targets, the PDA and a nearby display, are chosen from several so-called available endpoints: all the in-use end-points, two displays, and a fixed phone. What makes an end-point available depends on the business logic and it can vary from any known end-point that is currently registered with IMS to more complex criteria, such as being physically close to the user.

\section{Current State of the Art}

Different design aspects of SM have been investigated in the literature. Some of them are discussed in this section.

- Hiding the mobility: A number of projects choose to essentially hide the mobility of the user from the peer participants in the session by combining all the devices of the user as a special type of pseudo-device. The problem of SM becomes the problem of reconfiguring this meta-device. This can act as a gateway only for the SIP signalling traffic alone or for both SIP signalling and media traffic. In [8], the authors introduce the notion of virtual device as a set of local mobile devices that are actively discovered through SLP (Service Location Protocol). In the MUSIK framework [9] a multimedia gateway multiplexes or splits the media streams into flows distributed to the appropriate devices.

- Session mobility in ubiquitous computing: Beyond mechanisms to reconfigure media stream, SM is also an integration problem and ubiquitous-computing research tackled the problem from this integration point, albeit not in a SIP context. In [10], the authors make a distinction between a proactive handoff, where devices are involved in handling the transfer, and a reactive handoff where a server servicing an entire smart room or floor, identifies the target device and can intermediate the transfer. However ubiquitouscomputing research often considers non-SIP infrastructures built from scratch for a smart space. An example of work that does consider SIP, together with SLP for service discovery, is an architecture proposed in [11] which uses the location information and user preferences to increase the end-system capabilities and change device behaviour.

In a SIP/IMS context, the main researches related to SM are:

- SIP mechanisms: SIP has two schemes for SM between SIP devices, one is based on the REFER method and the other on the use of Third Party Call Controller (3PCC) [5]. A REFER method includes contact information of the corresponding end-point (e.g., a SIP URI) that the target end-point uses to re-INVITE it with an appropriate Replaces header. Alternatively, a 3PCC that also established the initial call among participants invites the target end-point and re-INVITEs the corresponding end-points in the conversation.

- User's context: The context of the user inherently plays an important role both in taking the decision to reconfigure a session and in actually implementing the SM support. Vorwerk et al. describe a media delivery framework that takes care of the changing user requirements to adapt the transport-overlay and implement transparent transcoding for a context-based transfer of a Video-on-Demand session between two devices [12].

- SIP-based mobility delays: The results of research on the delays of SIP-based handoff also apply, to a certain extent, to SM. Delays are incurred by the signalling delays during the reREGISTER phase and the re-INVITE phase, but the total delay to complete the entire handoff procedure, i.e., until the media streaming resumes, are higher. Delay-evaluation has been made both experimentally and analytically, through SIP-signalling models. Of 
particular interest is the evaluation of delays in heterogeneous networks for dual-interface terminals, for example UMTS to/from WLAN [13][14].

- Session mobility with IMS: With IMS, the mechanisms needed for SM can be built in a SIP application server (AS) [4]. A target device for a session transfer can be identified by a dedicated mobility server, or the triggering end-point itself can discover neighbouring endpoints. Mate et al. distinguish among device-centric mechanisms (everything is implemented in the devices), network-centric mechanisms (everything is implemented by the network), and hybrid architectures [6].

Researches do not converge to a specific SM solution. Furthermore, they propose non IMSbased SM solutions. However, IMS has been proposed by 3GPP to provide convergence. Hence, the next section discusses the options available to accommodate a session controller that facilitates SM within an IMS framework.

\section{SUPPORTING SESSION MOBILITY WITH IMS}

In implementing SM functionality and custom services that capitalize on it, one can identify several possible design choices. One is to build special-purpose code into all the participant SIP user agents, but this adds complexity to all the terminals. Another is to force all participants to first talk with a third-party server in order to participate in the session. This server would then implement all the functionality related to SM. Essentially, such an approach would be an extension of the current model of supporting audio conferences, where all participants dial a special number to register in the conference. This has the disadvantage of not being transparent to the user, who has to know the contact information of the server.

A third possibility, and the most natural to IMS, is to take advantage of the fact that, with IMS, all the SIP traffic goes through the S-CSCF (Serving Call/Session Control Function). The SCIM (Service Capability Interaction Manager) sub-component within the S-CSCF will first channel all the SIP traffic through the registered SIP ASs, before this traffic is forwarded to the intended recipients, and each SIP AS can modify it according to its internal logic. Consequently, all the functionality related to SM can be at the level of a SIP AS (see figure 2) acting as a Session Controller (SC) in a manner that is transparent to both the served end-points and the other corresponding end-points. This controller also intercepts HTTP traffic issued by the served endpoints (see figure 2). It is not unusual for both cellular operators and wired ISPs to set up an HTTP proxy for the clients connecting to their network.

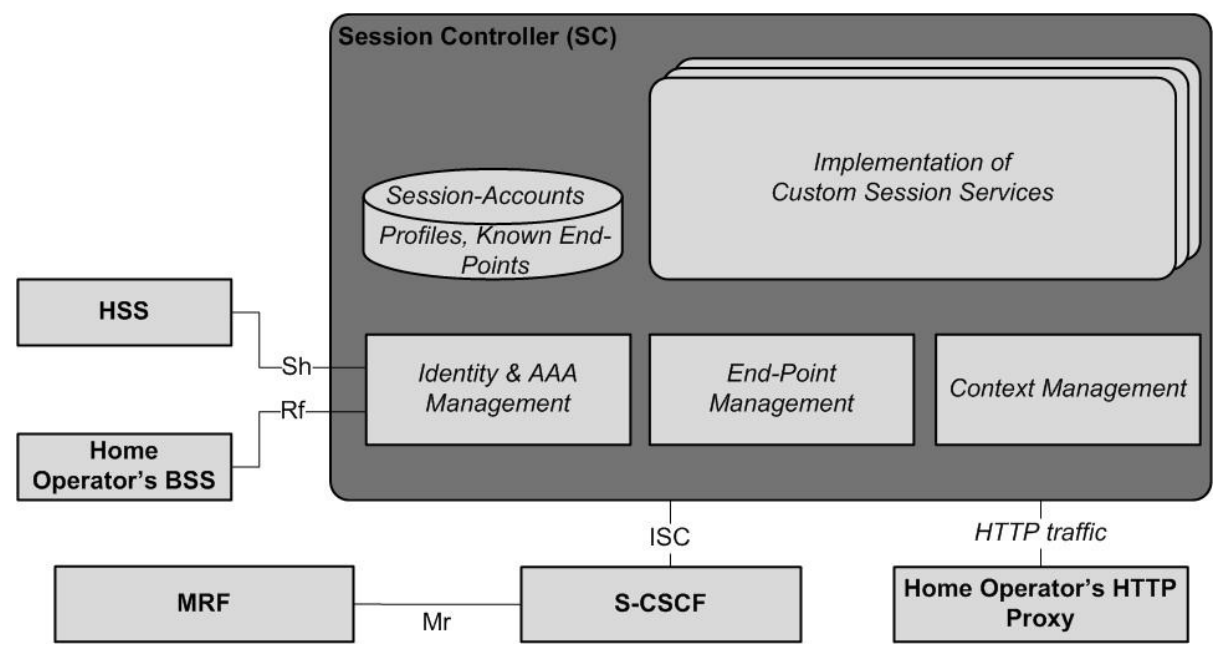

Figure 2. Session Mobility with a SIP AS: components and mechanisms 
The International Journal of Multimedia \& Its Applications (IJMA) Vol.3, No.2, May 2011

One of the challenges in implementing mechanisms for SM is to identify the commonalities across multiple application domains, in order to devise a generic session manager or reusable building blocks. As a step towards this goal, figure 2 identifies several functional components needed to support SM and outlines how they interface to standard IMS components. The remainder of this section discusses each of these components.

\subsection{Functional Components}

The main role of the controller is to form and manage a consistent view of the state of the session associated with each account and to assist custom modules in reconfiguring this session according to their internal logic.

\section{- Custom Session Services}

Each such module implements a custom service providing added-value functionality related to the mobility of the session. The implementation of the custom session-services must not require changes to the typical IMS clients representing the corresponding end-points. In fact, for the interactions needed by many custom session-services, the capabilities of typical IMS clients might also suffice for the known end-points. The main task of each particular session-service is to determine when to trigger a SM procedure and to which target devices the selected on-going communication should be redirected.

\section{- Session Accounts}

The custom session-services are enabled selectively for each session-account. Each account has an associated profile. In addition to the list of activated custom session-services, this profile also contains any other settings and user preferences specific to each of these services, configurable or not by human users. It also contains the list of end-points known for the account and potentially their characteristics and capabilities. For a residentialaccount example, the associated known end-points could be:

○ the cellular phone or the PDA of each family member,

○ the TV set-top-box in the living room, in mode "all ages watching",

$\circ$ the soft IMS client of each family member when logged-in on the PC in the study room and the one in the basement,

$\circ$ the fixed SIP phones in the basement and in the living room.

Other information already available in the home subscriber server (HSS) or accessible through a GUP (General User Profile) Server can also be considered part of the account profile.

\section{- End-Point Management:}

While the account-session profile includes static information about the known end-points, an end-point management component is needed to keep track of the in-use and of the available end-points for each session-account. Not all the known end-points are available at all times. Consider the example of the residence account and the set-top-box in the living room. The set-top-box can be configured in two modes, one that corresponds to the all ages watching, and one that corresponds restricted watching. Each mode corresponds to a different end-point, and the two cannot be available at the same time. More advanced implementations can also use context information to decide if an end-point should be considered available or not, and further trim down and customize the list of available endpoints.

In addition to maintaining the available and in-use lists, the end-point management also maintains a list of corresponding end-points for the session. It also keeps track of on-going communications between in-use end-points and the corresponding end-points. All this is 
The International Journal of Multimedia \& Its Applications (IJMA) Vol.3, No.2, May 2011

achieved by analyzing the messages exchanged in the SIP INVITE transactions, SIP REGISTER transaction, and BYE messages, as well as by analyzing the communication established over HTTP. An essential responsibility of the end-point management component is to implement the mechanisms for SM and to carry on session reconfigurations, at the request and under the control of the custom session-services activated for the account.

\section{- Identity AAA Management:}

Seamless authorization and authentication is needed in the process of exchanging reINVITE and REFER SIP messages and this falls under the responsibility of the Identity \& AAA Management component. One way to uniquely identify known end-points could be by a pair IMPI - IMPU (IMS Private Identity - IMS Public Identity). This would identify the device or pseudo device through the IMPI, and users using the device(s) through the IMPU. The Identity \& AAA Management component cooperates with the HSS (Home Subscriber Server) over the $S h$ reference point to carry on its function. To assist the custom sessionservices in carrying on their charging strategy, the Identity \& AAA Management component also interfaces with the Home Operator's Billing Support System (BSS) over the Rf reference point for off-line charging.

\section{- Context Management:}

This component is in charge of obtaining, keeping up to date, and communicating contextual information about the in-use/available end-points and their human users to the custom session-services and the other components of the Session Controller, while hiding the particular details of how and where from the information was obtained. Each context item, depending on its type and frequency of change, can be either accessed on demand, i.e., the interested component polls the context management component for the data it needs, or it can be made available through a publish-subscribe mechanism, with the interested component subscribing to be notified of changes.

In the example of residence account, the location of end-points, as given by the device from the location server of the cellular operator, is an instance of contextual information. Other examples are the technical characteristics end-points, as stored by the HSS or by a GUP server, or as determined from the signalling traffic.

\subsection{Session Controller Connectivity}

In figure 2, the SC connects with other components in the operator's network as is described hereafter.

\section{- $S C-S-C S C F$ :}

The ISC is reference point between the SC and S-CSCF as defined in [1]. Over this reference point passes the SIP traffic to/from the UE. All other SIP traffic issued by the SC, for instance to a presence server or to the MRF (Media Resource Function), traverses the same reference point. The MRF can provide to the SC and to the custom session-services centralized media processing capabilities such as conferencing, transcoding and prompt/collect. $M r$ reference point between the S-CSCF and the MRF is SIP-based and is not yet fully standardized by $3 \mathrm{GPP}$. An IETF draft issued on November 18, 2006, and expired on May 22, 2007 proposes a SIP interface to Voice XML media services from the MRF, interface with similar functionality to the $M r$ reference point.

\section{- SC - HTTP proxy:}

On one hand, through the Home Operator's HTTP proxy, the SC can gain access to the HTTP exchanges to/from the server end-points. On the other hand, through the same HTTP 
The International Journal of Multimedia \& Its Applications (IJMA) Vol.3, No.2, May 2011

proxy (or through another special purpose HTTP proxy), the SC can access certain IMS servers/services in the network of the Home Operator, such as a presence server or a GUP server, over HTTP. Likely the HTTP access will be secured with TLS protocol, and the proxy can also serve as an authentication proxy, similar to the scheme proposed for the $U t$ reference point of UE by the 3GPP specification TS 33-22.

\section{- $S C$ - HSS:}

The SC interacts with the HSS, the repository of user-related information, over the Sh reference point. Based on the Diameter protocol described in the 3GPP specification TS 29328 , the $S h$ reference point serves to download and update subscriber data. It also allows subscriptions to HSS-data changes. The information stored at the HSS includes: IP multimedia private identities (IMPIs) and IP multimedia public identities (IMPUs) for the subscribers, services and application servers, requested data/domain, IMS registration state, and domain information. It can also include custom data. In fact the entire session-account profile can be stored in the HSS.

\section{- $S C-B S S$ :}

The SC also interacts with Home operator's BSS for offline charging through Diameter $R f$ interface. It sends accounting information to a Charging Collector Function (CCF) located in the same administrative domain. Based on the collected information, the CCF builds a CDR (Call Detail Record) which is sent to the Billing System (BS) of the domain. Each session has a unique IMS Charging Identifier (ICI) and an Inter-Operator Identifier (IOI) which defines the originating and terminating networks. Each domain has its own BS which is generally a proprietary system. Details on the Diameter $R f$ reference point can be found in 3GPP specification TS 32-225 and TS 32-299.

The SM described above is supported at the network layer. During the transfer, a session is not seen as an ongoing communication at the network layer. Hence, appropriate resource reservation should be defined to give SM arrival calls the required priority and guarantee better user experience. Such an approach is proposed in the following section.

\section{SESSION MOBILITY RESOURCE ALLOCATION}

Several research studies have addressed the radio resource allocation issues in wireless networks and their impact on the QoS, defined as the blocking probability of new and handoff calls [15][16][17]. Call blocking is one of the main significant performance measures in wireless networks. Specifically, it is necessary to control the blocking of handoff calls which result in the interruption of a connection in a mid of an ongoing communication thus yielding to a bad QoS experience. Different resource allocation strategies have been proposed. They can be classified into two types: with or without priority. Moreover, the radio resource assignment could be static or dynamic and with or without calls queuing [18][19][20][21][22][23].

Comparative studies of these strategies reveal that radio resource reservation giving priority to handoff calls allows reducing the blocking probability of such calls. Moreover, improvement is possible with handoff calls queuing support.

In this paper, we propose to use static radio resource reservation without queuing to serve the new, the handoff and the SM arrival calls in a mobile network. The main reasons for such a choice are the radio resource allocation approach simplification and the impact analysis of radio resource reservation on new, handoff and SM calls' blocking. The resulting resource reservation approach gives higher priority to handoff and SM calls compared to new calls. We refer to it as three dimensional resource allocation approach (3D approach). We also propose to compare this approach with that giving priority only to handoff calls and handling the SM calls with the same 
priority as new calls. We refer to this approach as two dimensional resource allocation approach (2D approach).

To detail these approaches, we define two decision thresholds $C-C_{1}$ and $\mathrm{C}-\mathrm{C}_{2}$. As soon as $\mathrm{C}_{-} \mathrm{C}_{1}$ radio resources are in use by ongoing communications for both approaches, new calls are blocked. SM calls are also blocked when $\mathrm{C}_{-} \mathrm{C}_{1}$ radio resources are in use by ongoing communications for the 2D approach.

For the 3D approach:

- If handoff calls have greater priority than SM calls, then the latter calls are blocked when $\mathrm{C}-\mathrm{C}_{2}$ radio resources are in use by ongoing communications, and handoff calls are blocked when all resources are used by ongoing communications.

- Otherwise, SM calls are blocked when all resources are in use by ongoing communications, and handoff calls are blocked when $\mathrm{C}-\mathrm{C}_{2}$ radio resources are in use by ongoing communications.

For the 2D approach, handoff calls are blocked when all resources are in use by ongoing communications. Using these approaches, we propose analytical models to compute the blocking probability of the new, handoff and SM calls for both approaches. These models are described in the following section.

\section{BLOCKING PROBABILITY ANALYSIS}

We propose to use a multidimensional birth-death process to model the two approaches where the arrivals of the different calls are assumed to be independent and their processes are Poisson. The derived models are used to compute the blocking probability for the two approaches.

\subsection{D approach}

This section describes the analytical model for the 2D approach and gives the blocking probability computation details of the new, the SM and the handoff calls.

\subsubsection{D approach}

The radio cell state is defined as follows

$$
e=(i ; j)
$$

where $i$ is the number of new and SM calls that arrived in the radio cell at an instant $t, 0 \leq i \leq C$ $-C_{1}$, and $j$ is the number of handoff calls that arrived in the radio cell at an instant $t, 0 \leq j \leq C$.

The radio resource allocation procedure in a radio cell operates according to the following rules:

- new and SM calls are blocked when $i=\left(C-C_{l}\right)$ or $i+j=C$,

- handoff calls are blocked only when $i+j=C$,

where $0 \leq C_{1} \leq C$. Let $\lambda_{n s}$ be the new and the SM call arrival rate and $\lambda_{h}$ the handoff call arrival rate. The new and the SM call duration follow an exponential law with mean $1 / \mu_{n s}$. Furthermore, the handoff call duration follows an exponential law with mean $1 / \mu_{h}$.

We have $A_{n s}=\lambda_{\mathrm{ns}} / \mu_{\mathrm{ns}}, A_{n s}=\lambda_{\mathrm{h}} / \mu_{\mathrm{h}}, A=A_{n s}+A_{h}, A_{h}=\beta A$ and $A_{n s}=(1-\beta) A$ ( $\beta$ represents the ratio $A_{h}$ to $\left.A\right)$. Figure 3 shows a transition diagram from/to state $(i ; j)$ to/from four neighbor states. 


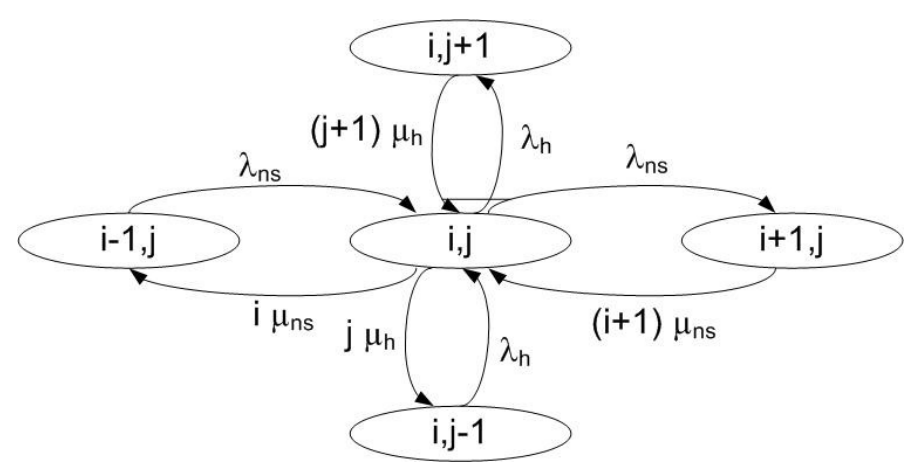

Figure 3. Transition diagram for the 2D approach

For $0 \leq j \leq C$ and $0 \leq i \leq C-C_{1}$, the steady state equations are given by:

where

$$
\left(\lambda_{h}+\lambda_{n s}+j \mu_{h}+i \mu_{n s}\right) P(i ; j)=Z
$$

$$
Z=\mu_{n s}(i+1) P(i+1 ; j)+\mu_{h}(j+1) P(i ; j+1)+\lambda_{h} P(i ; j-1)+\lambda_{n s} P(i-1 ; j) .
$$

We verify a posteriori that the solution $P(i, j)=\left(A_{n s}^{i} / i !\right)\left(A_{h}^{j} / j !\right)$ satisfies equation (3). This yields to a solution that is the product of two one-dimensional birth-death processes which are independent. This solution can be generalized for approaches with more than two dimensions such as the 3D approach. Thereby, a solution that is a product of three one-dimensional and independent birth-death processes exists for the 3D approach. With $i$ and $j$ ongoing communications in a radio cell, the associated stationary probability is given by:

$$
P(i, j)=\frac{A^{(i+j)}}{K} \frac{(1-\beta)^{i}}{i !} \frac{\beta^{j}}{j !}
$$

with $0 \leq i \leq C-C_{1}, 0 \leq j \leq C-i$ and the normalization constant $K$ is given by :

$$
K=\sum_{i=0}^{C-C_{1} C-i} \sum_{j=0}^{(i+j)} \frac{(1-\beta)^{i}}{i !} \frac{\beta^{j}}{j !}
$$

\subsubsection{Call Blocking Probability}

We compute the blocking probability of new and SM call arrivals, $P_{b, n s}$, and of handoff call arrivals, $P_{b, h}$.

Figure 4 shows an example of the different translations between the states of the $2 \mathrm{D}$ approach where $C=5$ and $C_{l}=2$. 


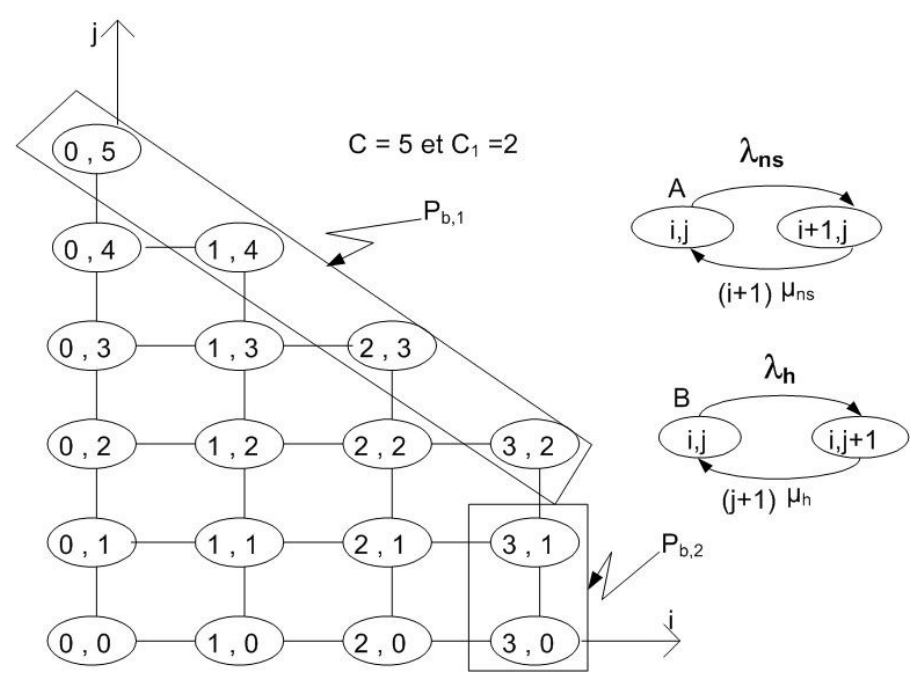

Figure 4. Call blocking probability of the 2D approach

In this figure, $\mathrm{P}_{\mathrm{b}, 1}$ represents the blocking probability of handoff calls when $i+j=C . \mathrm{P}_{\mathrm{b}, 2}$ is the blocking probability of new and SM calls when $i=C-C_{l}$ and $j<C_{l}$. The blocking probability $P_{b, h}$ is given by :

$$
\begin{aligned}
P_{b, h} & =P_{b, 1}=P\left(i+j=C, C_{1} \leq j \leq C\right) \\
& =\sum_{j=C_{1}}^{C} \frac{A^{C-j}(1-\beta)^{C-j} A^{j} \beta^{j}}{K j !(C-j) !)}=\frac{A^{C}}{K}\left[\frac{1}{C !}-\sum_{j=0}^{C_{1}-1} \frac{(1-\beta)^{C-j} \beta^{j}}{j !(C-j) !}\right]
\end{aligned}
$$

The blocking probability $P_{b, n s}$ is given by:

$$
\begin{aligned}
P_{b, n s} & =P_{b, 1}+P\left(i=C-C_{1}, j \leq C_{1}\right) \\
& =P_{b, 1}+\sum_{j=0}^{C_{1}-1} \frac{A^{C-C_{1}}(1-\beta)^{C-C_{1}} A^{j} \beta^{j}}{K j !\left(C-C_{1}\right) !} \\
& =P_{b, 1}+\frac{(A(1-\beta))^{C-C_{1}}}{K\left(C-C_{1}\right) !} \sum_{j=0}^{C_{1}-1} \frac{A^{j} \beta^{j}}{j !}
\end{aligned}
$$

Hence, we have

$$
P_{b, n s}-P_{b, h}=\frac{[A(1-\beta)]^{C-C_{1}}}{\left(C-C_{1}\right) !} \sum_{j=0}^{C_{1}-1} \frac{(A \beta)^{j}}{j !}
$$

From equation (8), we note that $P_{b, n s} \geq P_{b, h}$. Moreover, when $C_{1}=0$, we have $P_{b, n s}=P_{b, h}$ $=\operatorname{Erlang} B(A ; C)$.

\subsection{D approach}

This section describes the analytical model of the 3D approach. It details the blocking probability computation of the new, SM and handoff calls. 
Parameters $C_{1}$ and $C_{2}$ allow defining the following radio resource allocation policy when either of the handoff or SM calls have higher priority compared to the other calls. In the following, we describe the methodology and the analytical model for the blocking probability computation when handoff calls have higher priority than the SM and new calls. These method and model are similar to the case when the SM calls have higher priority than the handoff and new calls. In this case, references to and symbols of the SM are interchanged with those of the handoff calls. Specifically, the policy is given by the following rules:

- new calls are blocked when $i=\left(C-C_{1}\right)$ or $i+j+k=C$;

- $\mathrm{SM}$ calls are blocked when $j=\left(C-C_{2}\right)$ or $i+j+k=C$;

- handoff calls are blocked when $i+j+k=C$.

The SM and handoff call arrivals are characterized by Poisson processes with exponential durations.

\subsubsection{Model}

We define the radio cell state as follows:

$$
e=(i ; j ; k)
$$

where $i$ is the number of new calls, $0 \leq i \leq C-C_{1}, j$ is the number of SM calls, $0 \leq j \leq C$ $-C_{2}$, and $k$ is the number of handoff calls, $0 \leq k \leq C$. These call arrivals are independent and follow a Poisson law. Let $\lambda_{n}$ be the new call arrival rate, $\lambda_{s}$ the SM call arrival rate and $\lambda_{h}$ the handoff call arrival rate. The new, the SM and handoff call durations follow an exponential law with mean $1 / \mu_{n}, 1 / \mu_{s}$ and $1 / \mu_{h}$ respectively. Hence, we have $A_{n}$ $=\lambda_{n} / \mu_{n}, A_{s}=\lambda_{s} / \mu_{s}, A_{h}=\lambda_{h} / \mu_{h}$ and $A=A_{n}+A_{s}+A_{h}$. The state transition diagram is given in figure 5 .

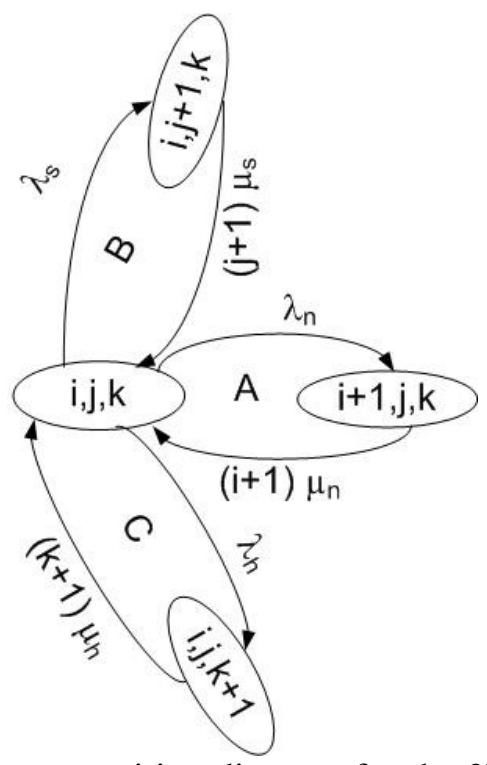

Figure 5. State transition diagram for the 3D approach

From this diagram, we obtain:

$$
P(i, j, k)=\frac{A_{n}^{i}}{i !} \frac{A_{s}^{j}}{j !} \frac{A_{h}^{k}}{k !} P(0,0,0)
$$


The probability $P(0 ; 0 ; 0)=K^{-1}$ is computed using the following normalization equations for $\left(C_{1}+C_{2}\right) \leq C$ and $\left(C_{1}+C_{2}\right)>C$ :

- Case 1: $\left(C_{1}+C_{2}\right) \leq C$

$$
\begin{aligned}
\sum_{i=0}^{C_{2}} & \sum_{j=0}^{C-C_{2}} \sum_{k=0}^{C-i-j} P(i, j, k)+\sum_{i=C_{2}+1}^{C-C_{1}} \sum_{j=0}^{C-i} \sum_{k=0}^{C-i-j} P(i, j, k)=1 \\
K & =\sum_{i=0}^{C_{2}} \sum_{j=0}^{C-C_{2} C-i-j} \sum_{k=0}^{C_{2}} \frac{A_{n}^{i}}{i !} \frac{A_{s}^{j}}{j !} \frac{A_{h}^{k}}{k !}+\sum_{i=C_{2}+1}^{C-C_{1}} \sum_{j=0}^{C-i} \sum_{k=0}^{C-i-j} \frac{A_{n}^{i}}{i !} \frac{A_{s}^{j}}{j !} \frac{A_{h}^{k}}{k !} \\
& =\sum_{i=0}^{C_{2}} \sum_{j=0}^{C-C_{2}} \sum_{k=0}^{C-i-j} A^{(i+j+k)} \frac{W^{i}}{i !} \frac{\beta_{1}^{j}}{j !} \frac{\beta_{2}^{k}}{k !}+\sum_{i=C_{2}+1}^{C-C_{1}} \sum_{j=0}^{C-i} \sum_{k=0}^{C-i-j} A^{(i+j+k)} \frac{W^{i}}{i !} \frac{\beta_{1}^{j}}{j !} \frac{\beta_{2}^{k}}{k !}
\end{aligned}
$$

- $\quad$ Case 2: $C_{1}+C_{2}>C$

$$
\begin{gathered}
\sum_{i=0}^{C-C_{1}} \sum_{j=0}^{C-C_{2} C-i-j} \sum_{k=0} P(i, j, k)=1 \\
K=\sum_{i=0}^{C-C_{1} C-C_{2} C-i-j} \sum_{j=0}^{\sum_{k=0}} \frac{A_{n}^{i}}{i !} \frac{A_{s}^{j}}{j !} \frac{A_{h}^{k}}{k !}=\sum_{i=0}^{C-C_{1} C-C_{2} C-i-j} \sum_{j=0} \sum_{k=0}^{(i+j+k)} \frac{W^{i}}{i !} \frac{\beta_{1}^{j}}{j !} \frac{\beta_{2}^{k}}{k !}
\end{gathered}
$$

With $A_{s}=\beta_{1} A, A_{h}=\beta_{2} A$ and $W=1-\beta_{1}-\beta_{2}$, the probability $P(i ; j ; k)$ is given by:

$$
P(i, j, k)=\frac{A^{(i+j+k)}}{K} \frac{\left(1-\beta_{1}-\beta_{2}\right)}{i !} \frac{\beta_{1}^{j}}{j !} \frac{\beta_{2}^{k}}{k !}
$$

\subsubsection{Call blocking probability}

Three blocking probabilities are defined:

- new call blocking probability $P_{b, 1}$ when $i=C-C_{1}, j \leq C_{1}$ and $k<C_{1}-j$,

- SM call blocking probability $P_{b, 2}$ when $j=C-C_{2}, i \leq C_{2}$ and $k<C_{2}-i$,

- handoff call blocking probability $P_{b, 3}$ when $i+j+k=C, i \leq C-C_{1}$ and $j \leq$ $\left(C-C_{2}-i\right)$.

These probabilities are computed for both cases: $C_{1}+C_{2} \leq C$ and $C_{1}+C_{2}>C$. Figure 6 shows these probabilities. 

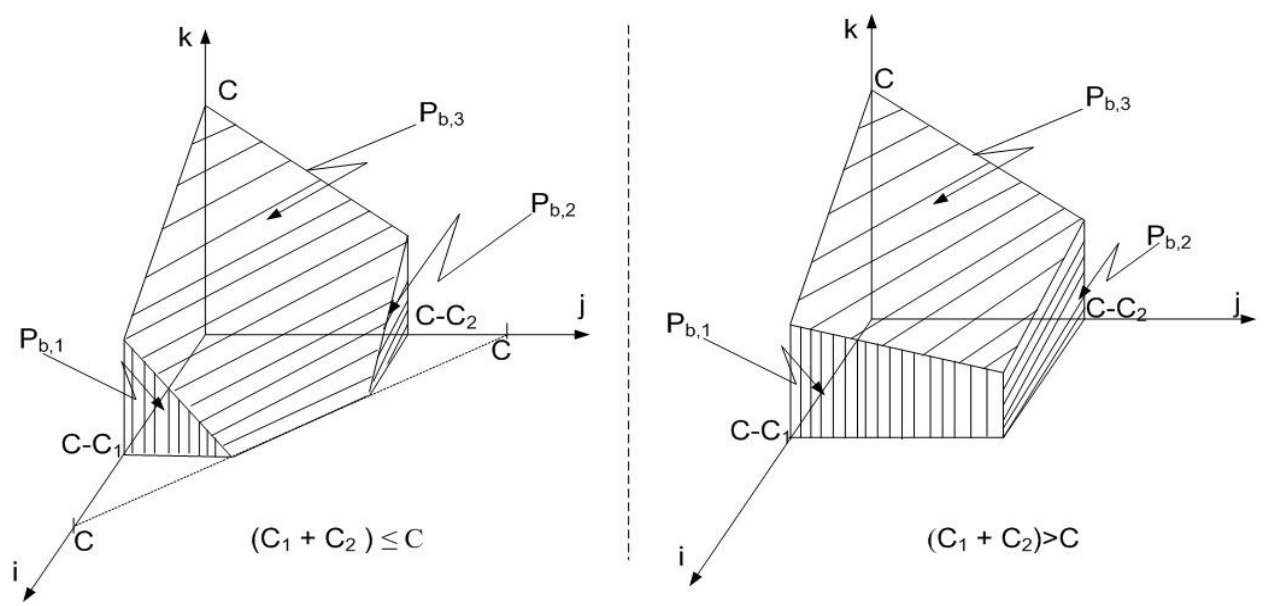

Figure 6. Call blocking probabilities for the 3D approach

For $C_{1}+C_{2} \leq C, P_{b, h}$ can be expressed as:

$$
P_{b, h}=P_{b, 3}=\frac{A^{C}}{K} \sum_{i=0}^{C_{2}} \sum_{j=0}^{C-C_{2}} \frac{W^{i}}{i !} \frac{\beta_{1}^{j}}{j !} \frac{\beta_{2}^{C-(i+j)}}{(C-(i+j)) !}+\frac{A^{C}}{K} \sum_{i=C_{2}+1}^{C-C_{1}} \sum_{j=0}^{C-i} \frac{W^{i}}{i !} \frac{\beta_{1}^{j}}{j !} \frac{\beta_{2}^{C-(i+j)}}{(C-(i+j)) !}
$$

where $P_{b, 3}=P\left(i+j+k=C\right.$; $\left.0 \leq i \leq C-C_{1} ; 0 \leq j \leq C-C_{2}-i\right) . P_{b, s}$ is given by:

$$
\begin{aligned}
P_{b, s}=P_{b, 3}+P_{b, 2} & =P_{b, 3}+P\left(j=C-C_{2}, 0 \leq i \leq C_{2}, 0 \leq k \leq C_{2}-i\right) \\
& =P_{b, 3}+\frac{\left(A \beta_{1}\right)^{\left(C-C_{2}\right)}}{K\left(C-C_{2}\right) !} \sum_{i=0}^{C_{2}} \sum_{k=0}^{C_{2}-i} \frac{A^{(i+k)} W^{i}}{i !} \frac{\beta_{2}^{k}}{k !}
\end{aligned}
$$

We also have

$$
\begin{aligned}
P_{b, n}=P_{b, 3}+P_{b, 1} & =P_{b, 3}+P\left(i=C-C_{1}, 0 \leq j \leq C_{1}, 0 \leq k \leq C_{1}-j\right) \\
& =P_{b, 3}+\frac{(A W)^{\left(C-C_{1}\right)}}{K\left(C-C_{1}\right) !} \sum_{j=0}^{C_{1}} \sum_{k=0}^{C_{1}-j} \frac{A^{(j+k)} \beta_{1}^{j}}{j !} \frac{\beta_{2}^{k}}{k !}
\end{aligned}
$$

For $C_{1}+C_{2}>C, P_{b, n}$ represents the blocking probability for new calls when $i=C-C_{1}$, $j \leq C-C_{2}$ and $k<C_{1}-j . P_{b, s}$ is the blocking probability of the SM call arrivals when $j$ $=C-C_{2}, i \leq C-C_{1}$ and $k<C_{2}-i . P_{b, h}$ is the blocking probability of the handoff call arrivals when $i+j+k=C, i \leq C-C_{1}$ and $j \leq C-C_{2}$. These probabilities are given by:

$$
P_{b, h}=P_{b, 3}=\frac{A^{C}}{K} \sum_{i=0}^{C-C_{1} C-C_{2}} \sum_{j=0} \frac{W^{i}}{i !} \frac{\beta_{1}^{j}}{j !} \frac{\beta_{2}^{C-(i+j)}}{(C-(i+j)) !}
$$

where $P_{b, 3}=P\left(i+j+k=C ; 0 \leq i \leq C-C_{1} ; 0 \leq j \leq C-C_{2}\right)$,

$$
P_{b, s}=P_{b, 3}+P_{b, 2}=P_{b, 3}+\frac{\left(A \beta_{1}\right)^{\left(C-C_{2}\right)}}{K\left(C-C_{2}\right) !} \sum_{i=0}^{C-C_{1} C_{2}-i} \sum_{k=0}^{A^{(i+k)} W^{i}} \frac{\beta_{2}^{k}}{i !}
$$


where $P_{b, 2}=P\left(j=C-C_{2} ; 0 \leq i \leq C-C_{1} ; 0 \leq k \leq C_{2}-i\right)$, and

$$
P_{b, n}=P_{b, 3}+P_{b, 1}=P_{b, 3}+\frac{(A W)^{\left(C-C_{1}\right)}}{K\left(C-C_{1}\right) !} \sum_{j=0}^{C-C_{2} C_{1}-j} \sum_{k=0}^{\frac{A^{(j+k)}}{\beta_{1}^{j}}} \frac{\beta_{2}^{k}}{k !}
$$

with $P_{b, 1}=P\left(i=C-C_{1} ; 0 \leq j \leq C-C_{2} ; 0 \leq k \leq C_{1}-j\right)$.

\section{NUMERICAL RESULTS}

The call blocking probability for the $2 \mathrm{D}$ and $3 \mathrm{D}$ approaches dependent on the total number of radio resources $C$ available in a radio cell, the variables $\mathrm{C}_{1}$ and $\mathrm{C}_{2}$, and the offered traffics $A$, $A_{n s}, A_{n}, A_{h}$ and $A_{s}$.

Figure 7 shows the new, the SM and the handoff call blocking probability variations for the 2D approach. It shows the evaluated values from the proposed blocking probability computation model and the values obtained by simulations. These results show that when $C_{l}$ increases the new and the SM call blocking probability increases, while the handoff blocking probability decreases. Note that the simulation results are similar to those obtained with the call blocking probability computation model. The small difference between the results obtained by simulation and those obtained by the proposed model is due to the error generated by the simulator.

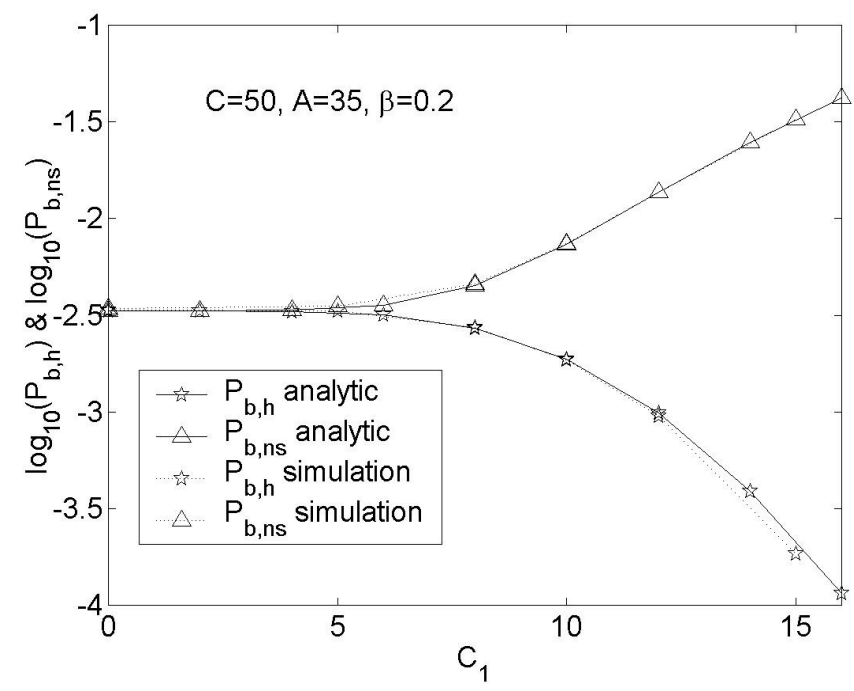

Figure 7. New, session mobility and handoff blocking probabilities of the 2D approach

The probability values are summarized in Table 1 . These results show that the higher $C_{l}$ is the higher are the new and the SM blocking probabilities. These probabilities may be reduced by increasing the number of radio resources $C$ in a radio cell. The example given in Table 1 shows that by increasing the value of $C$ to 100 , the new and the SM blocking probability becomes of the order of $10^{-4}$ rather than $10^{-3}$ for $C=50$. However, increasing $C$ to reduce the blocking probability of the SM calls is a costly solution. Thereby, we propose to use the 3D approach to give higher priority to the SM calls compared to the new calls and even the handoff calls if required by the network operator and the service quality constraints. 
Table 1. Call blocking probabilities for the 2D model

\begin{tabular}{|c|c|c|c|c|c|}
\hline \multirow[t]{2}{*}{$\mathrm{C}_{1}$} & \multicolumn{2}{|c|}{$\begin{array}{c}C=50, A=35 \text { and } \\
\beta=0.2\end{array}$} & \multicolumn{3}{|c|}{$C=100, A=70$ and $\beta=0.25$} \\
\hline & $P_{b, h}\left(x 10^{-3}\right)$ & $P_{b, n s}\left(x 10^{-3}\right)$ & $\mathbf{C}_{1}$ & $P_{b, h}\left(\times 10^{-3}\right)$ & $P_{b, n s}\left(\times 10^{-3}\right)$ \\
\hline 0 & 3.33324 & 3.33324 & 16 & 0.13629 & 0.14116 \\
\hline 2 & 3.33260 & 3.33379 & 18 & 0.13263 & 0.15206 \\
\hline 4 & 3.31448 & 3.35284 & 20 & 0.12411 & 0.18712 \\
\hline 6 & 3.17424 & 3.54684 & 22 & 0.10870 & 0.28178 \\
\hline 8 & 2.70414 & 4.49754 & 24 & 0.08674 & 0.50372 \\
\hline 10 & 1.86904 & 7.36557 & 26 & 0.06165 & 0.96887 \\
\hline 12 & 0.98688 & 13.64230 & 28 & 0.03841 & 1.85991 \\
\hline 14 & 0.38923 & 24.81207 & 30 & 0.02078 & 3.44282 \\
\hline 16 & 0.11489 & 42.14727 & 32 & 0.00971 & 6.07239 \\
\hline
\end{tabular}

Figure 8 gives an example of the new, the SM and the handoff call blocking probabilities of the 3D approach for $C=50$ and when $C_{1}+C_{2} \leq C$. The new call blocking probability increases when $\mathrm{C}_{1}$ increases. The SM and the handoff call blocking probabilities decrease when $C_{l}$ increases. The obtained values of these probabilities are summarized in tables II and III for different values of $C_{1}, C_{2}$ and $C$.

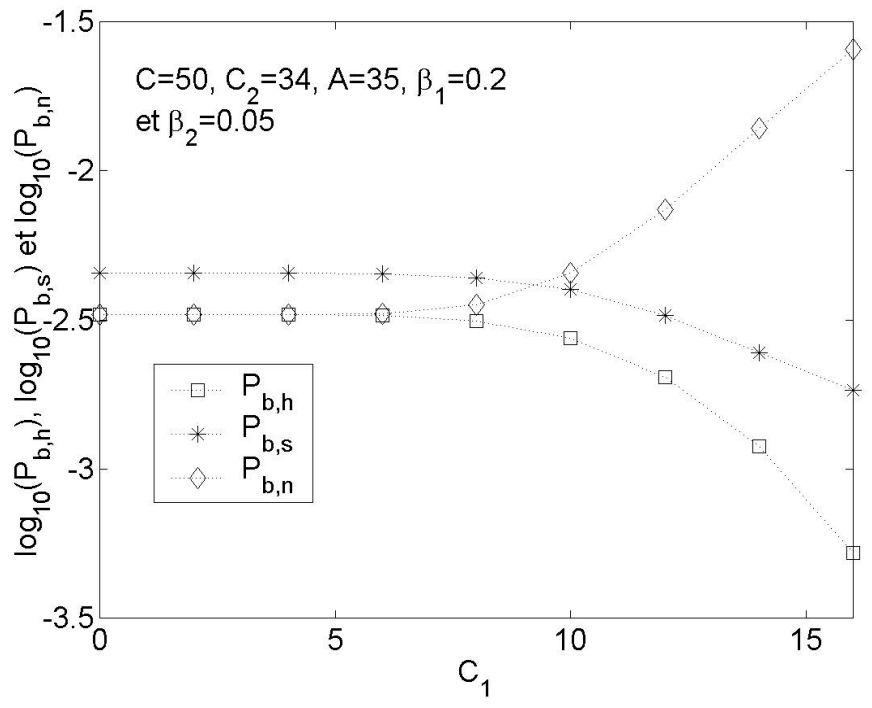

Figure 8. New, session mobility and handoff blocking probabilities of the 3D approach

Table 2 shows that smaller $C_{2}$ is the closer is the handoff blocking probability to that of the SM calls. This is true because the smaller $C_{2}$ is the closer is the SM blocking threshold $C-C_{2}$ to $C$. For a fixed $\mathrm{C}_{2}$ value, we notice that the higher $\mathrm{C}_{1}$ is the smaller are the blocking probabilities of the session and handoff calls. Moreover, for $C=100$, these probabilities are of the order of $10^{-4}$ while the new call blocking probability is of the order of $10^{-3}$ (table 3 ). 
Table 2. Call blocking probabilities of the 3D approach for $C_{1}+C_{2} \leq C$ with $C_{2}$ variable

\begin{tabular}{|c|c|c|c|}
\hline \multirow{2}{*}{$\mathbf{C}_{\mathbf{2}}$} & \multicolumn{3}{|c|}{ Call blocking probability $\left(\mathbf{x 1 0} \mathbf{- 3}^{-\mathbf{3}}\right)$} \\
\cline { 2 - 4 } & $\mathbf{C = 5 0 ,} \mathbf{A = 3 5}, \mathbf{C}_{\mathbf{1}}=\mathbf{2 4}, \boldsymbol{\beta}_{\mathbf{1}}=\mathbf{0 . 2}$ and $\boldsymbol{\beta}_{\mathbf{2}}=\mathbf{0 . 0 5}$ \\
\cline { 2 - 4 } & $\mathbf{P}_{\mathbf{b}, \mathbf{h}}$ & $\mathbf{P}_{\mathbf{b}, \mathbf{s}}$ & $\mathbf{P}_{\mathbf{b}, \mathbf{n}}$ \\
\hline 12 & 0.00227 & 0.00227 & 146.30792 \\
\hline 14 & 0.00227 & 0.00227 & 146.30792 \\
\hline 16 & 0.00227 & 0.00227 & 146.30792 \\
\hline 18 & 0.00227 & 0.00227 & 146.30792 \\
\hline 20 & 0.00227 & 0.00227 & 146.30792 \\
\hline 22 & 0.00227 & 0.02278 & 146.30792 \\
\hline 24 & 0.00227 & 0.00228 & 146.30792 \\
\hline 26 & 0.00227 & 0.00248 & 146.30792 \\
\hline
\end{tabular}

Table 3. Call blocking probabilities of the 3D approach for $C_{1}+C_{2} \leq C$ with $C_{1}$ variable

\begin{tabular}{|c|c|c|c|c|c|c|c|}
\hline \multirow[t]{3}{*}{$C_{1}$} & \multicolumn{7}{|c|}{ Call blocking probability $\left(\times 10^{-3}\right)$} \\
\hline & \multicolumn{3}{|c|}{$\begin{array}{l}C=50, A=35, C_{2}=34, \\
\beta_{1}=0.2 \text { and } \beta_{2}=0.05\end{array}$} & \multicolumn{4}{|c|}{$\begin{array}{c}C=100, A=70, C_{2}=68, \beta_{1}=0.2 \\
\text { and } \beta_{2}=0.05\end{array}$} \\
\hline & $\mathbf{P}_{b, \mathbf{h}}$ & $\mathbf{P}_{\mathrm{b}, \mathbf{s}}$ & $\mathbf{P}_{\mathrm{b}, \mathbf{n}}$ & $\mathrm{C}_{1}$ & $\mathbf{P}_{\mathbf{b}, \mathbf{h}}$ & $\mathbf{P}_{\mathrm{b}, \mathbf{s}}$ & $\mathbf{P}_{b, \mathbf{n}}$ \\
\hline 0 & 3.287 & 4.528 & 3.287 & 16 & 0.136 & 0.150 & 0.140 \\
\hline 2 & 3.287 & 4.528 & 3.287 & 18 & 0.132 & 0.146 & 0.151 \\
\hline 4 & 3.286 & 4.527 & 3.289 & 20 & 0.123 & 0.137 & 0.186 \\
\hline 6 & 3.264 & 4.505 & 3.319 & 22 & 0.108 & 0.122 & 0.281 \\
\hline 8 & 3.137 & 4.379 & 3.543 & 24 & 0.086 & 0.100 & 0.503 \\
\hline 10 & 2.747 & 3.990 & 4.525 & 26 & 0.061 & 0.075 & 0.968 \\
\hline 12 & 2.030 & 3.281 & 7.425 & 28 & 0.038 & 0.052 & 1.859 \\
\hline 14 & 1.189 & 2.458 & 13.869 & 30 & 0.020 & 0.034 & 3.442 \\
\hline 16 & 0.523 & 1.833 & 25.591 & 32 & 0.009 & 0.023 & 6.072 \\
\hline
\end{tabular}

Tables 4 and 5 give an example of the new, the SM and the handoff blocking probabilities for the 3D approach when $C_{1}+C_{2} \leq C$ and $C_{1}+C_{2} \geq C$.

Table 4. Call blocking probabilities of the $3 \mathrm{D}$ approach for $C_{1}+C_{2} \leq C$ with different $\beta_{1}$ and $\beta_{2}$ values

\begin{tabular}{|c|c|c|c|c|c|c|}
\hline \multirow[t]{3}{*}{$\mathbf{C}_{1}$} & \multicolumn{6}{|c|}{$\begin{array}{c}\text { Call blocking probability }\left(\mathrm{x10}^{-3}\right) \\
\mathrm{C}=50, \mathrm{~A}=35, \mathrm{C}_{2}=40,\end{array}$} \\
\hline & \multicolumn{3}{|c|}{$\beta_{1}=0.05$ and $\beta_{2}=0.2$} & \multicolumn{3}{|c|}{$\beta_{1}=0.2$ and $\beta_{2}=0.05$} \\
\hline & $\mathbf{P}_{\mathbf{b}, \mathbf{h}}$ & $\mathbf{P}_{\mathbf{b}, \mathbf{s}}$ & $\mathbf{P}_{\mathbf{b}, \mathbf{n}}$ & $\mathbf{P}_{\mathbf{b}, \mathbf{h}}$ & $\mathbf{P}_{\mathbf{b}, \mathbf{s}}$ & $\mathbf{P}_{\mathbf{b}, \mathbf{n}}$ \\
\hline 10 & 2.793 & 2.805 & 4.899 & 2.793 & 73.500 & 4.899 \\
\hline 12 & 2.077 & 2.089 & 7.903 & 2.077 & 73.190 & 7.903 \\
\hline 14 & 1.237 & 1.249 & 14.286 & 1.237 & 73.410 & 14.286 \\
\hline 16 & 0.573 & 0.586 & 25.848 & 0.573 & 75.074 & 25.848 \\
\hline 18 & 0.205 & 0.218 & 44.204 & 0.205 & 79.307 & 44.204 \\
\hline
\end{tabular}


Table 5. Call blocking probabilities of the 3D approach for $C_{1}+C_{2} \geq C$ with different $\beta_{1}$ and $\beta_{2}$ values

\begin{tabular}{|c|c|c|c|c|c|c|}
\hline \multirow{2}{*}{$\mathbf{C}_{1}$} & \multicolumn{5}{|c|}{$\begin{array}{c}\text { Call blocking probability } \\
\mathbf{C}=\mathbf{5 0}, \mathbf{A}=\mathbf{3 5} \mathbf{C}_{\mathbf{2}}=\mathbf{4 0}\end{array}$} \\
\cline { 2 - 7 } & \multicolumn{5}{|c|}{ ) } \\
\cline { 2 - 7 } & \multicolumn{2}{|c|}{$\boldsymbol{\beta}_{\mathbf{1}}=\mathbf{0 . 0 5}$ and $\boldsymbol{\beta}_{\mathbf{2}}=\mathbf{0 . 2}$} & \multicolumn{2}{|c|}{$\boldsymbol{\beta}_{\mathbf{1}}=\mathbf{0 . 2}$ and $\boldsymbol{\beta}_{\mathbf{2}}=\mathbf{0 . 0 5}$} \\
\cline { 2 - 7 } & $\mathbf{P}_{\mathbf{b}, \mathbf{h}}$ & $\mathbf{P}_{\mathbf{b}, \mathbf{s}}$ & $\mathbf{P}_{\mathbf{b}, \mathbf{n}}$ & $\mathbf{P}_{\mathbf{b}, \mathbf{h}}$ & $\mathbf{P}_{\mathbf{b}, \mathbf{s}}$ & $\mathbf{P}_{\mathbf{b}, \mathbf{n}}$ \\
\hline 10 & 2.793 & 2.804 & 4.570 & 1.551 & 79.778 & 3.881 \\
\hline 12 & 2.077 & 2.088 & 7.468 & 0.789 & 79.306 & 7.049 \\
\hline 14 & 1.237 & 1.248 & 13.907 & 0.209 & 78.903 & 13.646 \\
\hline 16 & 0.573 & 0.585 & 25.620 & 0.029 & 78.764 & 25.505 \\
\hline 18 & 0.205 & 0.218 & 44.105 & 0.002 & 78.742 & 44.072 \\
\hline
\end{tabular}

From these tables, we notice that the handoff calls blocking probability is smaller than that of the SM calls and this despite the fact that $\beta_{2}>\beta_{1}$. However, for $\beta_{1}>\beta_{2}$, the SM blocking probability is higher than that obtained with $\beta_{1}<\beta_{2}$. Hence, according to the service need in mobile networks, it is possible to have:

- small handoff call blocking probability using the 3D approach for small and high handoff traffics $\left(\beta_{2}>\beta_{1}\right.$ and $\left.\beta_{2}<\beta_{1}\right)$ in both cases, i.e., when $C_{1}+C_{2} \leq C$ or $C_{1}+C_{2} \geq C$;

- $\quad$ small SM call blocking probability using the 3D approach for small SM traffics $\left(\beta_{1}<\beta_{2}\right)$ in both cases, i.e., when $C_{1}+C_{2} \leq C$ or $C_{1}+C_{2} \geq C$.

By interchanging the radio resource reservation such that the SM calls are blocked when $i+j+k=C$ and the handoff calls are blocked when $k=C-C_{2}$, it is possible to have low SM call blocking probability both for small and high traffics generated by such call arrivals in both cases, i.e., when $\mathrm{C}_{1}+\mathrm{C}_{2} \leq \mathrm{C}$ or $\mathrm{C}_{1}+\mathrm{C}_{2} \geq \mathrm{C}$.

\section{Conclusion}

While SM has been studied in both SIP and non-SIP contexts, IMS integration issues are often left aside. Mechanisms to support SM are introduced by IETF RFCs but wider integration in IMS is not considered by 3GPP, ETSI, ITU, and TISPAN standards. This article gave an overview of the functionality needed and the issues associated with supporting context-based SM within the IMS framework. The design of a Session Controller SIP AS was explored. Such a controller can enable the development of custom session-services that implement scenarios of seamless SM to better adapt to the changing user context.

Since session transfer is performed at the application level, the resulting arrival call could be considered as a new one in the network level rather than an ongoing communication. Consequently, higher priority should be given to the SM arrival than to the new call arrival. Hence, this paper proposed to use a priority-based radio resource allocation approach to support SM in converged networks. A new analytical model that gives different priority levels to three types of call arrivals: new, SM and handoff was developed. In addition to this model, called 3D model, we presented a 2D analytical model that gives priority only to handoff calls. Based on these models, the call blocking probabilities have been computed for different offered traffics and different values of radio resource available in a radio cell. Numerical results showed that the proposed 3D model, compared to the 2D model, allows achieving lower blocking probabilities for the SM and the handoff calls compared to new calls. Moreover, depending on the priority policy set, low call blocking probabilities can be achieved for different call arrival rates. We are in the process of implementing several such services as an effective demonstration of 
The International Journal of Multimedia \& Its Applications (IJMA) Vol.3, No.2, May 2011

convergence in wireless and wired IMS networks for a better quality of experience for endusers.

\section{REFERENCES}

[1] 3GPP, "IP Multimedia Subsystem (IMS)," 3GPP TS 23.228, September 2007.

[2] J. Rosenberg, H. Schulzrinne, G. Camarillo, A. Johnston, J. Peterson, R. Sparks, M. Handley, and E. Schooler, "SIP: Session initiation protocol," RFC 3261, June 2002.

[3] O. Karsten, W. Stefan, and S. Arkadiusz, "Enhanced methods for SIP session mobility in a converged network," in 16th IST Mobile and Wireless Communications, July 2007, pp. 15.

[4] C. Yun, J. Park and Y. Lim, "Session mobilitybof IP Multimedia Subsystem (IMS) using modified assured session Transfer" in Proceeding of the $15^{\text {th }}$ Asia-Pacific Conference on Communications, October 2009.

[5] R. Shacham, H. Schulzrinne, S. Thakolsri, and W. Kellerer, "Session initiation protocol (SIP) session mobility", IETF RFC5631, October 2009.

[6] C. Huang and C. Tsai, "A SIP-based session mobility management framework for ubiquitous multimedia services", in Proceedings of the Fifth International Conference on Ubiquitous Intelligence and Computing (UIC08), 2008.

[7] 3GPP, "Feasibility study on multimedia session continuity," 3GPP TR 23.893, September 2007.

[8] R. Shacham, S. Schulzrinne, S. Thakolsri, and W. Kellerer, "The virtual device: expanding wireless communication services through service discovery and session mobility," in IEEE International Conference on Wireless And Mobile Computing, Networking And Communications, 2005. (WiMob'2005), vol. 4, 22-24 Aug. 2005, pp. 73-81.

[9] R. Kothari and A. Ganz, "Musik: multimedia service integration framework for smart environments," in Consumer Communications and Networking Conference, 2006. CCNC 2006. 2006 3rd IEEE, vol. 1, 8-10 Jan. 2006, pp. 203- 207.

[10] Y. Cui, K. Nahrstedt, and D. Xu, "Seamless user-level handoff in ubiquitous multimedia service delivery," Multimedia Tools Appl., vol. 22, no. 2, pp. 137-170, 2004.

[11] S. Berger, H. Schulzrinne, S. Sidiroglou, and X. Wu, "Ubiquitous computing using SIP," in NOSSDAV '03: Proceedings of the 13th international workshop on Network and operating systems support for digital audio and video. New York, NY, USA: ACM Press, 2003, pp. 82-89.

[12] M. Vorwerk, S. Schuetz, R. Aguero, J. Choque, S. Schmid, M. Kleis, M. Kampmann, and M. Erkoc, "Ambient networks in practice - instant media services for users on the move," in In Proc. of 2nd International Conference onTestbeds and Research Infrastructures for the Development of Networks and Communities, TRIDENTCOM 2006, Barcelona, Spain, March 2006, pp. 1-2.

[13] N. Banerjee,W.Wu, K. Basu, and S. K. Das, "Analysis of SIP-based mobility management in 4G wireless networks," Computer Communications, vol. 27, no. 8, pp. 697-707, 2004.

[14] W. Wu, N. Banerjee, K. Basu, and S. Das, "SIP-based vertical handoff between WWANs and WLANs," Wireless Communications, IEEE, vol. 12, no. 3, pp. 66-72, June 2005.

[15] D. Calin, "Mod'ele de trafic pour les r'eseaux mobiles multim'edia," Th`ese de Doctorat, D'epartement d'Informatique et R'eseaux, Universit'e de Versailles, July 1998.

[16] Chlamtac, A. Farag'o, T. Henk, and G. Gordos, "Optimizing bandwith allocation in cellular networks with multirate traffic," IEEE GLOBECOM', London, pp. 1126-1130, November 1996.

[17] J. Huard, "Handoff study in cellular network," Report, Electrical Engineering department, Columbia University, December 1993. 
The International Journal of Multimedia \& Its Applications (IJMA) Vol.3, No.2, May 2011

[18] Katzela and M. Naghshineh, "Channel assignment schemes for cellular mobile telecommunication systems: a comprehensive survey," IEEE Personal Communications, pp. 10-31, June 1996.

[19] J. Markoulidakis, G. Lyberopoulos, and M. Anagnostou, "Traffic model for third generation cellular mobile telecommunication systems," Wireless Networks, pp. 389-400, 1998.

[20] M. Marsan, S. Mrano, C. Mastroianni, and M. Meo, "Performance analysis of cellular mobile communication networks supporting multimedia services," ACM/Baltzer Journal on Mobile Networks and Applications (MONET), vol. 5, pp. 167-177, 2000.

[21] M. Oliver and J. Borr'as, "Performance evaluation of variable reservation policies for handoff prioritization in mobile networks," IEEE INFOCOM'99, vol. 9, pp. 1187-1194, 1999.

[22] M. Oliver and J. Borras, "Performance evaluation of variable reservation policies for handoff prioritization in mobile networks," IEEE INFOCOM'99, vol. 9, pp. 1187-1194, March 1999.

[23] S. Rappaport and C. Purzynski, "Prioritized resource assignment for mobile cellular communication systems with mixed services and platform types," IEEE Trans. Veh. Technol, vol. 45, no. 3, pp. 443-458, August 1996. 